\title{
Radiofrequency ablation for the treatment of stage I non-small cell lung cancer in high-risk patients
}

\author{
Arjun Pennathur, MD, ${ }^{a}$ James D. Luketich, MD, ${ }^{a}$ Ghulam Abbas, MD, ${ }^{a}$ Mang Chen, MD, ${ }^{a}$ Hiran C. Fernando, MD, ${ }^{c}$ \\ William E. Gooding, MS, ${ }^{b}$ Matthew J. Schuchert, MD, ${ }^{a}$ Sebastien Gilbert, MD, ${ }^{a}$ Neil A. Christie, MD, ${ }^{a}$ \\ and Rodney J. Landreneau, MDa
}

Earn CME credits at http://cme.ctsnetjournals.org
From the Heart, Lung, and Esophageal Surgery Institute, ${ }^{\text {a }}$ University of Pittsburgh Medical Center, and the University of Pittsburgh Cancer Institute Biostatistics Facility, ${ }^{\mathrm{b}}$ Pittsburgh, Pa; and Boston Medical Center, ${ }^{\mathrm{c}}$ Boston, Mass.

This research was funded in part by the National Institutes of Health (NIH) Specialized Program of Research Excellence in Lung Cancer (P50 CA090440) and in part by research grants from Boston Scientific, Inc, and RITA Medical, Inc.

James Luketich reports grant support from RITA Medical, and Hiran Fernando reports lecture fees from Boston Scientific.

Read at the Thirty-second Annual Meeting of the Western Thoracic Surgical Association, Sun Valley, Idaho, June 21-24, 2006.

Received for publication June 27, 2006; revisions received April 1, 2007; accepted for publication April 11, 2007.

Address for reprints: James D. Luketich, MD, Sampson Family Endowed Professor of Surgery, The Heart, Lung, and Esophageal Surgery Institute, University of Pittsburgh Medical Center, 200 Lothrop St, C-800, Pittsburgh PA 15213 (E-mail: luketichjd@upmc.edu).

J Thorac Cardiovasc Surg 2007;134:857-64 0022-5223/\$32.00

Copyright $(9) 2007$ by The American Association for Thoracic Surgery

doi:10.1016/j.jtcvs.2007.04.060
Objective: Surgical resection is the standard of care for stage I non-small cell lung cancer. The objective of this study was to evaluate computed tomography-guided radiofrequency ablation as an alternative treatment option for high-risk patients with stage I non-small lung cancer.

Methods: Patients with medically inoperable stage I non-small lung cancer were offered radiofrequency ablation. Thoracic surgeons evaluated and performed radiofrequency ablation under computed tomographic scanning guidance. Response was assessed by means of computed tomographic and positron emission tomographic scanning. Time to progression and survival were monitored every 3 months.

Results: Nineteen patients underwent radiofrequency ablation over a 3-year period. There were 8 men and 11 women with a median age of 78 years (range, 68-88 years). Radiofrequency ablation resulted in pneumothorax requiring a pigtail catheter in $12(63 \%)$ patients. An initial complete response was observed in $2(10.5 \%)$ patients, a partial response in $10(53 \%)$ patients, and stable disease in $5(26 \%)$ patients. Early progression occurred in $2(10.5 \%)$ patients. During follow-up, local progression occurred in $8(42 \%)$ nodules, and the median time to progression was 27 months. There were no procedure-related mortalities, and 6 deaths occurred during follow-up. The mean follow-up in the remaining patients was 29 months (range, 9-52 months). The probability of survival at 1 year was estimated to be $95 \%$ (95\% confidence interval, 0.85-1.0). The median survival was not reached.

Conclusion: Our experience indicates that radiofrequency ablation is safe in highrisk patients with stage I non-small lung cancer, with reasonable results in patients who are not fit for surgical intervention.

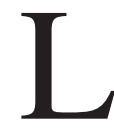
ung cancer is the most common cause of cancer-related mortality in the United States. Surgical resection is the standard treatment in resectable disease and offers the best chance of cure, particularly in the earlier stages. ${ }^{1-3}$ In an aging population, many patients with otherwise resectable lung cancer have other comorbidities, including pulmonary dysfunction, which might preclude them from surgical resection. ${ }^{4}$ In these patients conventional external beam radiotherapy is typically offered as treatment, with reported 5-year survival rates of $10 \%$ to $30 \% .^{5-8}$ Sibley and colleagues ${ }^{6}$ reviewed the results of radiotherapy for stage I non-small lung cancer (NSCLC) from Duke University in 156 patients and reported a 2- and 5-year survival of $39 \%$ and 13\%, respectively. Recently, Qiao and associates $^{8}$ reviewed 18 studies investigating the treatment of stage I NSCLC with radiotherapy and reported a mean 3-year and 5-year overall survival of 34\% and $21 \%$, respectively. Thus the results of conventional radiotherapy have not been satisfactory, prompting investigators to study other modalities of treatment, such as radiofrequency ablation (RFA), in this high-risk group of patients with lung cancer. 


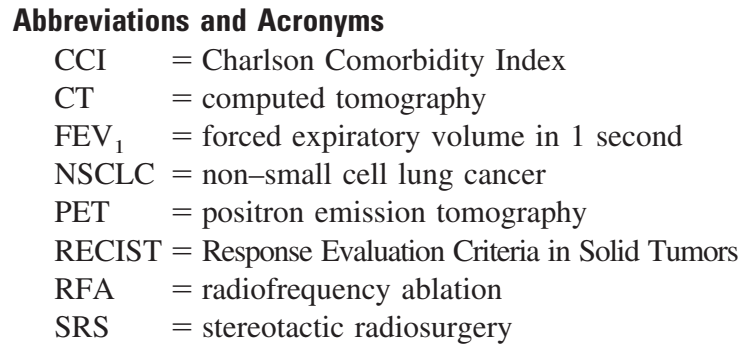

The use of interstitial hyperthermia to treat lung neoplasm was initially reported by Lilly and colleagues ${ }^{9}$ in 1983. RFA is a thermal ablative technique and is a relatively new modality of treatment, which might be applicable in high-risk patients with lung cancer. There have been several reports in the literature on the use of RFA for lung neoplasm, but many of these are case reports or series with a focus on immediate response, without rigorous longer-term follow-up for recurrence or survival. ${ }^{10-14}$ Furthermore, there are few reports with an emphasis on stage I NSCLC. We have previously described our experience with RFA in the treatment of both primary and metastatic lung neoplasms. ${ }^{15,16}$ The principal findings of our earlier report were that RFA was more effective for smaller $(\leq 5 \mathrm{~cm})$ tumors, with better early survival and response to treatment. Additionally, in our previous report, we described a modification of the Response Evaluation Criteria in Solid Tumors (RECIST) criteria (Table 1) that were used to assess treatment response and progression at the ablated sites. In this article we report our experience with the use of RFA in the treatment of stage I NSCLC in medically inoperable patients. This is part of an ongoing institutional review board-approved study that continues to accrue at the University of Pittsburgh.

\section{Materials and Methods}

We reviewed our experience with RFA for the treatment of stage I non-small lung neoplasm in medically inoperable patients at the University of Pittsburgh over a 3-year period from 2002-2005. Some of these patients have been reported previously. ${ }^{16}$ Informed consent was obtained from all patients, and the study was approved by the Institutional Review Board at the University of Pittsburgh.

\section{Selection of Patients}

Patients with NSCLC were routinely staged with chest computed tomographic (CT) scanning, and most patients also underwent a positron emission tomographic (PET) scan. Patients with mediastinal lymph nodes greater than $1 \mathrm{~cm}$ in the short axis, a positive PET scan result, or both underwent mediastinoscopy. Mediastinoscopy was performed in 2 patients, and left-sided video-assisted thoracoscopic surgery was performed in 1 patient for biopsy of hilar and aortopulmonary window nodes. The inclusion criteria for RFA in the treatment of patients with stage I NSCLC for this study were as follows: (1) patients who were considered medically inoperable because of poor pulmonary function, high cardiac risk, and/or other comorbidities and (2) presence of a target tumor of 4 $\mathrm{cm}$ or smaller. In addition, patients who refused an operation were offered RFA if the tumor was peripheral and less than $4 \mathrm{~cm}$. Exclusion criteria included central tumors. All patients were evaluated by a thoracic surgeon to determine inoperability and suitability for RFA.

\section{Treatment Protocol}

Technique. A percutaneous CT-guided approach was used in all patients, and as described previously, all procedures were performed by thoracic surgeons. ${ }^{15,16}$ The RFA equipment consists of a generator, active electrode, and dispersive pads. Electrosurgical dispersive pads (Dispersive Electrodes, RITA Medical Systems, Inc, Moutainview, Calif, or Valleylab Polyhesive, Valleylab, Boulder, Colo) were applied to the patient's thighs and plugged into the return electrode socket on the front panel of the radiofrequency generator.

RFA was performed by using 2 different radiofrequency generators and needle electrodes. The radiofrequency generator was set up in accordance with the generator's instructions for use. One

TABLE 1. Modified RECIST criteria

\begin{tabular}{|c|c|c|c|}
\hline Response & CT scan mass size & CT scan mass quality & PET scan* \\
\hline Complete (2 of the following) & $\begin{array}{l}\text { Lesion disappearance (scar) } \\
\text { or }<25 \% \text { original size }\end{array}$ & $\begin{array}{l}\text { Cyst cavity formation; } \\
\text { low density }\end{array}$ & SUV $<2.5$ \\
\hline Partial (1 of the following) & $\begin{array}{l}>30 \% \text { Decrease in the sum } \\
\text { LD of target lesions }\end{array}$ & $\begin{array}{l}\text { Mass central necrosis } \\
\text { or central cavity with } \\
\text { liquid density }\end{array}$ & $\begin{array}{l}\text { Decreased SUV or area } \\
\text { of FDG uptake }\end{array}$ \\
\hline $\begin{array}{l}\text { Stable lesion (1 of the } \\
\text { following) }\end{array}$ & $\begin{array}{l}<30 \% \text { Decrease in the sum } \\
\text { LD of target lesions }\end{array}$ & $\begin{array}{l}\text { Mass solid appearance, } \\
\text { no central necrosis } \\
\text { or cavity }\end{array}$ & $\begin{array}{l}\text { Unchanged SUV or } \\
\text { area of FDG uptake }\end{array}$ \\
\hline $\begin{array}{l}\text { Progression (2 of the } \\
\text { following) }\end{array}$ & $\begin{array}{l}\text { Increase of }>20 \% \text { in sum } \\
\text { LD of target lesions }\end{array}$ & $\begin{array}{l}\text { Solid mass, invasion } \\
\text { adjacent structures }\end{array}$ & $\begin{array}{l}\text { Higher SUV or larger } \\
\text { area of FDG uptake }\end{array}$ \\
\hline
\end{tabular}

CT, Computed tomography; PET, positron emission tomography; SUV, standardized uptake value of fluorodeoxyglucose F18; FDG, fluorodeoxyglucose F18; $L D$, lesion diameter. ${ }^{*}$ Positron emission tomographic scan done selectively. 
system comprised of a radiofrequency generator (RF3000; Boston Scientific Corp, Boston, Mass) and needle electrodes (LeVeen Needle Electrode; RadioTherapeutics Corporation, Sunnyvale, Calif). Under CT guidance, a finder needle (22-gauge long spinal needle) was used to determine the trajectory and placement of the active RFA probe. The LeVeen needle electrode (Boston Scientific Corp), which opens up as an array, was selected according to the diameter of the target lesion and placed into the target lesion. A 2-phase impedance-based algorithm was used according to the protocol suggested by the manufacturer. Briefly, the initial power applied was at the lowest setting and then increased in 5- to $10-\mathrm{W}$ increments until system impedance increased to more than $400 \Omega$. A second application of radiofrequency energy (a second phase) was provided at this same location (after waiting approximately 30 seconds) until system impedance increases to more than $400 \Omega$ for a second time.

The second system was comprised of a radiofrequency generator, the RITA Starburst XL Electrosurgical Device (RITA Medical Systems, Inc). Based on the size of the target tumor, the multitined expandable array (Starburst XL Electrosurgical Device, RITA Medical Systems, Inc) was deployed. Temperature was monitored from 5 electrodes, which are equipped with thermocouples. The radiofrequency generator was set to a target temperature of $90^{\circ} \mathrm{C}$, and the initial power was applied at between 35 and 50 W. The electrosurgical needle's deployment was staged according to the size of the tumor being treated, and the manufacturersuggested algorithm was followed.

With both of these systems, if necessary, the electrode was repositioned as many times as necessary to encompass the target tissue and a small rim with approximately 0.5 to $1 \mathrm{~cm}$ of nondiseased pulmonary tissue to ensure an adequate tumor margin.

Postprocedure follow-up of patients and assessment of response. Patients were followed up in 3-month intervals with clinical examinations, CT scans, and selectively with PET scans. Modified RECIST criteria were used to assess initial response to treatment at 3 to 5 months (Table 1). ${ }^{13,14}$ Patients were evaluated for initial response rate, time to local progression, and overall survival.

\section{Data Collection and Statistical Analysis}

The objective of the study was to determine the outcomes of RFA in the treatment of stage I NSCLC. Information on patient demographics, tumor characteristics, treatment, and comorbidities (Charlson Comorbidity Index [CCI]) were collected. ${ }^{17}$ Specific end points studied were complications, clinical response rates, time to local progression, and overall survival. The pretreatment CT scan was used as a baseline for evaluation of response and disease progression. Local disease progression of the treated nodule was assessed in accordance with the modified RECIST criteria in comparison with baseline diameter. The time to progression was calculated from the treatment date. Kaplan-Meier plots were constructed by using Greenwood confidence limits. The log-rank test was used to determine differences between groups. Association between categoric variables was tested with the Fisher exact test or the $\chi^{2}$ test.
TABLE 2. Patient characteristics

\begin{tabular}{lc}
\hline Sex (male/female) & $8 / 11$ \\
Median age (y) & 78 (range, 68-88) \\
Stage & 11 \\
IA & 8 \\
IB & \\
Staging procedures & 19 \\
CT scan & 15 \\
PET scan & 2 \\
Mediastinoscopy/VATS & \\
Histology & 8 \\
Squamous & 8 \\
Adenocarcinoma & 3 \\
Other & \\
Reason for RFA* & 10 \\
Poor PFT results & 7 \\
Increased cardiac risk & 8
\end{tabular}

CT, Computed tomography; PET, positron emission tomography; VATS, video-assisted thoracoscopic surgery; RFA, radiofrequency ablation; PFT, pulmonary function test. *Some patients had more than 1 reason for radiofrequency ablation.

\section{Results}

Nineteen patients underwent RFA over a 3-year period. There were 8 men and 11 women with a median age of 78 years (range, 68-88 years). There were 11 patients with stage IA and 8 with stage IB NSCLC. The mean size of the lesion was $2.6 \mathrm{~cm}$ (range, $1.6-3.8 \mathrm{~cm}$ ). These patients had significant comorbidities with the CCI, mean score being 5.5 (range, 3-12; median, 4). The most common reason for RFA was poor pulmonary function test results precluding resection. The median forced expiratory volume in 1 second $\left(\mathrm{FEV}_{1}\right)$ in these patients was 0.66 (29\% of predicted value), with a mean $\mathrm{FEV}_{1}$ of $0.73 \pm 0.21$. Patient characteristics and other reasons for RFA are summarized in Table 2.

The median hospital stay was 3 days (range, 1-7 days). The most common complication was pneumothorax requiring a pigtail catheter in $12(63 \%)$ patients. Prolonged air leak ( $>5$ days) occurred in $1(5 \%)$ patient. There were no procedure-related mortalities. Initial response was determined by using the modified RECIST criteria (Table 1). An initial complete response was observed in $2(10.5 \%)$ patients. A partial response was observed in $10(53 \%)$ patients. In these patients size criteria were met in 5 patients, and in the remaining 5 patients, a combination of decrease in metabolic activity on PET scanning, cavitation in the lesion, or both was used to determine partial response. All 3 criteria were met in 1 patient, 2 criteria were met in 5 patients, and 1 criterion (size $[\mathrm{n}=2]$, cavitation $[\mathrm{n}=1]$; low metabolic activity, standardized uptake value $=0.6$ on PET scan $[\mathrm{n}=1]$ ) was met in 4 patients. Stable disease was noted in $5(26 \%)$ patients. Early progression (at the time of assessment of initial response) occurred in 2 (10.5\%) patients. 


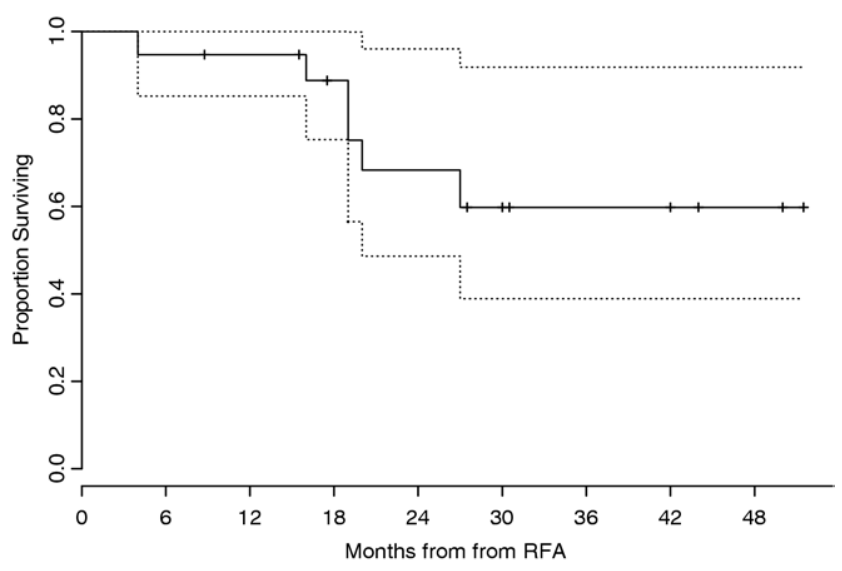

Figure 1. Kaplan-Meier plot showing the overall survival for the entire group with confidence limits. The time shown in the $x$-axis is in months from radiofrequency ablation (RFA). The dotted lines are $95 \%$ confidence bands for the probability of overall survival.

There were no procedure-related mortalities. There were 6 deaths during follow-up; 3 were cancer related, and 2 were not cancer related. One patient had distant recurrence, and the exact cause of death was not determined. The mean follow-up in the remaining patients was 29 months (median, 28 months; range, 9-52 months). The probability of overall survival at 1 year and 2 years were estimated to be $95 \%$ (95\% confidence interval, $85 \%-100 \%$ ) and $68 \%$ (95\% confidence interval 49\%-96\%), respectively. The median survival was not reached (Figure 1).

During follow-up, local progression, as determined by using the modified RECIST criteria, occurred in 8 (42\%) nodules, and the median time to progression was 27 months. Local progression was confirmed in $3(16 \%)$ patients by means of biopsy. Patients with locally progressive disease were treated with repeat RFA or stereotactic radiosurgery (SRS)/external beam radiation. One patient underwent resection. Patients with distant recurrences were treated with systemic therapy. No significant differences were noted in the time to progression and survival when patients with stage IA and IB disease were compared.

\section{Discussion}

Surgical intervention is the treatment of choice for resectable lung cancer and offers the best chance for cure. ${ }^{1-3} \mathrm{In}$ the medically inoperable patients with lung cancer, there are few effective options. Some of these patients with other associated comorbidities refuse treatment or receive no treatment with poor results. McGarry and coworkers ${ }^{18}$ reviewed the outcomes in 49 patients who received no treatment in early-stage disease, and the median survival was 14 months. Progressive cancer was the cause of death in 53\% of patients. Conventional radiotherapy, as described earlier, is offered to many of these patients, and the results again are not encouraging. ${ }^{5-8}$ Therefore in this high-risk patient population with lung cancer, newer modalities, such as RFA or SRS, might be applicable. ${ }^{19-21}$ Timmerman and associates ${ }^{20}$ reported the results in 37 patients with stage I NSCLC treated with SRS. The disease-free and overall survivals at a median follow-up of 15 months were 50\% and 64\%, respectively. We have presented our early results in patients with stage I NSCLC treated with SRS with a 91\% one-year overall survival at a median follow-up of 15 months. ${ }^{21}$ Further prospective studies are required to define the role of RFA and SRS in the treatment of lung neoplasms.

The issue of determination of medical operability or inoperability is critically important and should be assessed by a thoracic surgeon. One factor alone, such as poor pulmonary function test results, might not make a patient inoperable. With the potential benefits of lung volume reduction surgery, selected patients with upper lobe-predominant emphysema who have a coexistent lung cancer in the upper lobe may be candidates for lung resection, even when the pulmonary function test results are marginal. Choong and colleagues $^{22}$ reported a series of 21 patients with clinical stage I NSCLC with a mean $\mathrm{FEV}_{1}$ of 0.7 (29\% of predicted value) who underwent surgical resection. Lobectomy alone was performed in 9 patients, and in the remaining patients lobectomy or sublobar resection was supplemented with lung volume reduction surgery. In patients with pathologic stage I disease, the overall survival was $100 \%, 79 \%$, and $68 \%$ at 1,3 , and 5 years, respectively. Therefore the assessment of medical operability requires a comprehensive evaluation of not only pulmonary function but also other factors and comorbidities in the patient by a qualified thoracic surgeon.

We have previously reported the results of RFA in the treatment of both primary and metastatic lung cancer in inoperable high-risk patients. ${ }^{15,16}$ There have been very few reports of patients with stage I NSCLC who have been treated with RFA. Lee and associates ${ }^{11}$ reported their experience in 10 patients with stage I NSCLC. Of these 10 patients, only 4 were considered high-risk patients in whom surgical intervention was contraindicated, and the remainder refused surgical intervention. Mean survival in these 10 patients was reported to be 21 months, and $80 \%$ were alive at a mean follow-up of 14.8 months. Lencioni and cowork$\mathrm{ers}^{23}$ presented their preliminary results in 14 patients with stage I disease. Kaplan-Meier analysis showed an overall 1 -year survival of $81 \%$ at a mean follow-up of 9 months. Recently, Dupuy and colleagues ${ }^{24}$ reported their experience with RFA followed by external beam radiation in the treatment of stage I NSCLC. At a mean follow-up of 26 months, $14(58 \%)$ patients had died, and the estimated overall survival was $83 \%$ and $50 \%$ at 1 and 2 years, respectively. Our results of $95 \%$ overall survival in 1 year compare favorably with the results of these studies. 
These results during intermediate-term follow-up are encouraging, and the results appear to be equivalent or superior to the reported results with conventional radiation therapy. ${ }^{5-8}$ However, longer follow-up for this cohort is required, and full evaluation of survival end points will require greater maturity of time-to-event data. In addition, further prospective studies are required in high-risk patients to definitively compare RFA with conventional external beam radiation treatment or other emerging technologies, such as SRS.

This group of patients had significant associated comorbidities, with a mean Charlson score of 5.5 and a median CCI of 4 . The CCI was originally described to assess the effect of comorbidity on survival in 559 hospitalized patients. Nineteen conditions were found to significantly influence survival in the study population, and a weighted score was given based on the relative risk. ${ }^{17}$ This score has been validated in surgically resected patients with NSCLC in a study of 205 patients. ${ }^{25}$ The score was divided into 4 grades of increasing severity of the CCI index, with greater than 5 representing the highest grade of comorbidities. Multivariate analysis showed that a CCI 3 to 4 was the only predictive factor of increased risk of major complications (odds ratio, 9.8; 95\% confidence interval, 2.1-45.9), and for every increase in grade, the relative risk of an adverse outcome showed an increase. In the current study patients who underwent RFA were elderly (median age, 78 years), had significant comorbidities (mean CCI, 5.5), and represent a high-risk population.

The assessment of response after RFA is difficult because, unlike surgical resection, there is a lesion or scar that remains after therapy. There is considerable variation in how response is defined and evaluated. Chest CT scans, changes in contrast enhancement, and PET scans have all been used. Thus the reported response rate in the literature varies considerably. We have adopted strict criteria and have used modified RECIST criteria to evaluate response in these patients after RFA. We have combined not only the size of the lesion but also the changes in the density of the lesion along with metabolic activity based on PET scans to determine the response rate. The proposed modified RECIST criteria have limitations and have to be validated in a larger group of patients. Ultimately, however, disease progression and survival will be the measures by which the efficacy of RFA will have to be evaluated.

There are several factors that influence local recurrence or progression of disease. The important technical issues include (1) the degree of ablation and whether complete ablation is achieved and (2) the adequacy of margins of ablation obtained around the tumor. Completeness of ablation has also been evaluated in a few ablate-and-resect studies examining the extent of ablation after RFA. Review of these studies shows that effective $100 \%$ ablation varies from $38 \%$ to $67 \%$. Yang and coworkers ${ }^{26}$ presented the results of a multicenter ablate-and-resect study in 13 patients. The median tumor kill was $70 \%$, and $7(55 \%)$ patients had $100 \%$ ablation. These investigators also demonstrated a learning curve that exists in achieving 100\% ablation. Nguyen and associates ${ }^{27}$ did a prospective ablateand-resect study after open thoracotomy in patients with stage I or II NSCLC. RFA of the tumor was performed after a standard thoracotomy, and subsequently, a lobectomy was performed. Tumor cell viability was determined by means of routine histology, as well as supravital dye staining. Three (38\%) of 8 patients had complete ablation of tumors. In another study by Ambrogi and colleagues, ${ }^{28}$ a total of 9 patients underwent RFA either by means of the CT-guided approach or by means of open thoracotomy followed by resection. ${ }^{28}$ Complete ablation was noted in $6(67 \%)$ of 9 patients. However, supravital dye was not used to determine cell viability. The margins of ablation were a mean of $8 \mathrm{~mm}$ in completely ablated lesions and less than $5 \mathrm{~mm}$ in patients who had an incomplete ablation.

The adequacy of margins of ablation obtained around the tumor might be an important factor in local progression of disease. Our data with regard to wedge resections suggest a significant increase in the local recurrence rates $(14.6 \%$ vs $7.5 \%$ ) when the margins were less than $1 \mathrm{~cm}$ versus more than $1 \mathrm{~cm} .{ }^{29}$ In general, we strive to attain a $0.5-$ to $1-\mathrm{cm}$ margin around the tumor. Despite these margins, local progression (as determined by imaging studies) occurred in $42 \%$ of patients, and the median time to progression was 27 months. However, despite this incidence of progression, the results in terms of overall survival appear reasonable at intermediate-term follow-up. It is possible that the early detection of recurrence by means of close follow-up and the prompt treatment of recurrence might have contributed to the results of overall survival, but the limited number of patients did not allow us to derive definitive conclusions. In the future, further advances in technology or adjuvant therapy might be useful in decreasing this progression rate and perhaps in improving survival.

\section{Conclusions}

In summary, this study is a preliminary report on the use of RFA for the treatment of stage I NSCLC in medically inoperable patients. The results with regard to overall survival seem reasonable, with an estimated 1-year overall survival rate of $95 \%$. There are, however, several factors that merit further investigation, including optimal patient selection for RFA and the role of adjuvant therapy. Surgical resection is the standard treatment for stage I NSCLC in operable patients ${ }^{1-3}$; however, RFA might have a role in patients who are medically inoperable. Prospective studies are necessary to address these issues and to define the role of RFA in the treatment of lung neoplasms. Thoracic surgeons should continue to evaluate new technology and add 
these techniques to their armamentarium in the treatment of lung neoplasms. In conclusion, RFA appears safe in highrisk patients with stage I NSCLC, with reasonable results in patients who are not fit for surgical intervention.

\section{References}

1. Ginsberg RJ, Martini N. non-small lung cancer/surgical management In: Pearson FG, Cooper JD, Deslauriers J, et al, editors. Thoracic surgery. 2nd ed, Philadelphia: Churchill Livingstone; 2002. p. 837-59.

2. Ginsberg RJ, Rubinstein LV, Lung Cancer Study Group. Randomized trial of lobectomy versus limited resection for T1 N0 non-small lung cancer. Ann Thorac Surg. 1995;60:615-23.

3. Landreneau RJ, Sugarbaker DJ, Mack MJ, et al. Wedge resection versus lobectomy for stage I (T1 N0 M0) non-small-cell lung cancer. J Thorac Cardiovasc Surg. 1997;113:691-700.

4. Loran DB, Zwischenberger JB. Thoracic surgery in the elderly. $J$ Am Coll Surg. 2004;199:773-84.

5. Jeremic B, Classen J, Bamberg M. Radiotherapy alone in technically inoperable, medically inoperable, early stage (I/II) non-small lung cancer. Int J Radiat Oncol Biol Phys. 2002;54:119-30.

6. Sibley G, Jamieson T, Marks L, et al. Radiotherapy alone for medically inoperable stage I non-small-cell lung cancer: the Duke experience. Int J Radiat Oncol Biol Phys. 1998;40:149-54.

7. Kaskowitz L, Graham MV, Emami B, et al. Radiation therapy alone for stage I non-small lung cancer. Int J Radiat Oncol Biol Phys. 1993;27:517-23.

8. Qaio X, Tullgren O, Lax I, Sirzen F, Lewensohn. The role of radiotherapy in treatment of stage I non-small lung cancer. Lung Cancer. 2003:41:1-11.

9. Lilly MB, Brezovich IA, Atkinson W, et al. Hyperthermia with implanted electrodes: in vitro and in vivo correlations. Int J Radiat Oncol Biol Phys. 1983;9:373-82.

10. Dupuy DE, Zagoria RJ, Akerley W, Mayo-Smith WW, Kavanagh PV, Safran H. Percutaneous radiofrequency ablation of malignancies in the lung. AJR Am J Roentgenol. 2000;174:57-9.

11. Lee JM, Jin GY, Goldberg SN, et al. Percutaneous radiofrequency ablation for inoperable non-small lung cancer and metastasis: preliminary results. Radiology. 2004;230:125-34.

12. Akeboshi M, Yamakado K, Nakatsuka A, et al. Percutaneous radiofrequency ablation of lung neoplasms: initial therapeutic response. $J$ Vasc Interv Radiol. 2004;15:463-70.

13. Suh RD, Wallace AB, Sheehan RE, Heinze SB, Goldin JG. Unresectable pulmonary malignancies: CT-guided percutaneous radiofrequency ablation-preliminary results. Radiology. 2003;229:821-9.

14. Yasui K, Kanazawa S, Sano Y, et al. Thoracic tumors treated with CT-guided radiofrequency ablation: initial experience. Radiology. 2004;231:850-7.

15. Herrera LJ, Fernando HC, Perry Y et al. Radiofrequency ablation of pulmonary malignant tumors in nonsurgical candidates. $J$ Thorac Cardiovasc Surg. 2003;125:929-37.

16. Fernando HC, De Hoyos A, Landreneau RJ, et al. Radiofrequency ablation for the treatment of non-small lung cancer in marginal surgical candidates. J Thorac Cardiovasc Surg. 2005;129:639-44.

17. Charlson ME, Pompei P, Ales KL, et al. A new method of classifying prognostic comorbidity in longitudinal studies: development and validation. J Chronic Dis. 1987;40:373-83.

18. McGarry RM, Song G, DesRosiers P. Observation-only management of early stage, medically inoperable lung cancer. Chest. 2002;121: $1155-8$.

19. Whyte RI, Crownover R, Murphy MJ, et al. Stereotactic radiosurgery for lung tumors preliminary report of a phase I trial. Ann Thorac Surg. 2003;75:1097-101

20. Timmerman R, Papiez L, McGarry R, et al. Extracranial stereotactic radioablation results of a phase I study in medically inoperable stage I non-small lung cancer. Chest. 2003;124:1946-55.

21. Pennathur A, Luketich JD, Burton S, et al. Stereotactic radiosurgery for the treatment of lung neoplasm: initial experience. Ann Thorac Surg. 2007;83:1820-5.
22. Choong C, Meyers BF, Battafarano RJ, et al. Lung cancer resection combined with lung volume reduction in patients with severe emphysema. J Thorac Cardiovasc Surg. 2004;127:1323-31.

23. Lencioni RA, Crocetti L, Cioni R, et al. Percutaneous radiofrequency ablation of stage IA non-small cell lung cancer: a prospective multicenter clinical trial. J Clin Oncol. 2004;22:7175.

24. Dupuy DE, DiPetrillo T, Gandhi S, et al. Radiofrequency ablation followed by conventional radiotherapy for medically inoperable stage I non-small lung cancer. Chest. 2006;129:738-45.

25. Birim O, Maat APWM, Kappetein AP, Van Meerbeeck JP, Damhuis RAM, Bogers AJ. Validation of the Charlson comorbidity index in patients with operated primary nonsmall cell lung cancer. Eur J Cardiothorac Surg. 2003;23:30-4.

26. Yang S, Whyte R, Askin F, et al. Radiofrequency ablation of primary and metastatic lung tumors. Analysis of an ablate and resection study. Presented at: American Association for Thoracic Surgery 82nd Annual Meeting; 2002; Washington, DC.

27. Nguyen CL, Scott WJ, Young NA, Rader T, Giles LR, Goldberg M. Radiofrequency ablation of primary lung cancer: results from an ablate and resect pilot study. Chest. 2005;128:3507-11.

28. Ambrogi M, Fontanini G, Cioni R, et al. Biologic effects of radiofrequency thermal ablation on non-small lung cancer: results of a pilot study. J Thorac Cardiovasc Surg. 2006;131:1002-6.

29. El-Sherif A, Fernando HC, Santos R, Pettiford B, Luketich JD, Landreneau RJ. Margin and local recurrence after sublobar resection of NSCLC. Abstract presented at: Society of Surgical Oncology; 2005; Atlanta, Ga.

\section{Discussion}

Dr Donald E. Low (Seattle, Wash). Dr Millien, Dr Sintek, members, and guests, thank you very much. I appreciate the opportunity of discussing this article. I also would like to thank the authors for getting me the manuscript immediately before the meeting for review.

This article describes RFA treatment of 19 patients with stage I disease. All patients were reviewed by thoracic surgeons and were deemed inoperable on medical grounds. The authors should be congratulated in that surgeons not only carried out the assessment of these patients but also delivered the actual RFA therapy in the operating room. This is the third publication from your group examining RFA therapy in different patient populations. Your first study in 2003 examined 18 patients, 5 of whom had NSCLC. The next report in 2005 reported 18 patients, all of whom had NSCLC, but $50 \%$ had stage I disease. Clearly you are beyond the point of studying the feasibility of RFA in lung cancer and at a point where the focus should be on results, development of criteria for application of RFA, and, just as importantly, redefinition of criteria for medical inoperability. With that in mind, I believe that in a study of patients with true stage I disease, we should be very careful about opening the door to any nonsurgical approach, except in very carefully documented circumstances. Lung volume reduction surgery has taught us that pulmonary function tests are not the absolute limiting factor about who can undergo surgical intervention. Fifteen of 19 patients in your series were considered inoperable on the basis of "poor pulmonary function test results." However, the only objective indication you documented in your article was mean and median $\mathrm{FEV}_{1}$. Although it is reassuring that all of your patients were reviewed by thoracic surgeons, your current report would seem to provide excessive leeway for nonsurgeons to designate patient as nonsurgical candidates.

Do you not believe that at a minimum you should provide more specifics regarding predictable $\mathrm{FEV}_{1}$ and diffusion capacity in identifying persons who are inoperable on the basis of pulmonary 
function test results? In addition, should your conclusions not include a strong statement that short of being in a clinical trial, patients with stage I disease should always be seen by experienced thoracic surgeons and be presented in a multidisciplinary tumor board before being relegated to RFA therapy?

Dr Pennathur. Those are all valid points, and I think that one of the main issues regarding RFA is that there is not enough follow-up evaluating long-term results in the literature. In a recent review of the literature, most studies were case series with very poor follow-up and minimal information on how the recurrence was determined and the nature of the long-term results. That is one of the reasons why we wanted to and continue to study this more rigorously.

In terms of pulmonary function tests, our protocol comprises basically a predicted postoperative $\mathrm{FEV}_{1}$ value of less than $40 \%$ as one of the criteria. We use several criteria, with a complete assessment of the patient. Pulmonary function test results alone are not the sole criterion. The median $\mathrm{FEV}_{1}$ of these patients was 0.6, and these were elderly patients (median age, 78 years) who had multiple comorbidities. One of the measures of comorbidity that we assessed was the Charlson Comorbidity Score, which takes into consideration literally 19 conditions. It has been validated in several cancers and now has also been validated in lung cancer. In one study, in which the index was divided into categories of 0,1 to 2, 3 to 4, and the worst at greater than 5, an index of greater than 3 to 4 was shown to be significantly associated with an increase in postoperative complications. The mean Charlson Comorbidity Score in our group of patients as a whole was greater than 5. I share your concern that if these patients are not evaluated by thoracic surgeons and instead evaluated by others making the decision on inoperability, that is not right for the patient. As you pointed out, with the benefits of lung volume reduction surgery, these patients have to be evaluated by a thoracic surgeon, regardless of whether the patient is in a clinical trial, and we take into consideration not mere numbers but several other factors before we decide whether the patient is operable. In this series all patients were seen and evaluated by thoracic surgeons before performing RFA.

Dr Low. Just a comment before I go on to question 2, then. I would encourage you to solidify and make much more distinct the criteria that you are using for medical inoperability in your article.

The second question concerns the patient selection section of your article, which indicates that "most patients had PET scans." Do you think that PET scans should routinely be a component of the pretreatment assessment of these patients? Also, did all of these patients undergo pretreatment CT-guided biopsies to confirm a diagnosis before RFA therapy?

Dr Pennathur. Those are all excellent questions. This study went on over a period of $3 \frac{1}{2}$ years. Early on, PET scans were not routinely approved by insurance companies, and subsequently they were approved. Yes, I think a PET scan should be a part of the evaluation of these patients routinely. In this series $80 \%$ of the patients had a PET scan.

Regarding your second question, everybody had a CT-guided biopsy, and some of them were performed by the surgeon himself before the RFA. The tissue diagnosis was confirmed to be NSCLC in 18 of these patients, and in 1 patient it was called highly suspicious. Clinically, this patient had a suspicious lesion that was highly PET active, and with a suspicious call, we went ahead with RFA, but everybody else had a clear documentation of NSCLC.

Dr Low. Your report states that 6 patients died, with a median follow-up of 13.5 months. How many of these deaths were cancer related?

Dr Pennathur. That is again a good question. We have not actually analyzed that as cancer specific versus noncancer specific. Several of the patients did not die at the University of Pittsburgh, and on preliminary review and discussion with the primary physician, it appears that at least some of these were not cancer related, and we are in the process of ascertaining these data.

Dr Low. Your study demonstrates that pneumothoraces were immediate complications in $63 \%$ of your cases, although only 1 patient required a chest tube for greater than 4 days. Your main competition for localized nonsurgical treatment for stage I lung cancer is, of course, stereotactic radiation, which does not require any treatment-related intrathoracic instrumentation and can be used for more central tumors than those to which you have applied RFA in your series. Current reports of stereotactic radiation indicate local control rates of up to $80 \%$ to $90 \%$ at 1 and 2 years. Specifically, what do you see as the major advantages of RFA over stereotactic radiation?

I would like to thank the association for the opportunity to discuss this article.

Dr Pennathur. Again, an excellent point. I think that SRS and RFA could be potentially used in the same group of patients and in fact can be used in a complementary fashion. We do not use RFA for central lesions because of the proximity of the pulmonary artery and the bronchus, and therefore this is primarily applicable for peripheral lung tumors. Stereotactic radiosurgery (SRS) can be used for central lung lesions, although at a decreased dose. In terms of the analysis of the results of SRS, I have not found anything in the United States with long-term follow-up. That is one of the reasons we are studying SRS very closely. One of the few publications in the United States was by Dr Richard Whyte, a multicenter trial, and the other publication was from Dr Timmerman. He reported a total of 37 patients with stage I NSCLC, inoperable patients. Median follow-up was 14 months, and the 15 -month overall survival was $65 \%$; therefore I do not see the data as mature enough to comment on SRS in the United States. Similarly, we have also reported our initial experience with SRS with a median follow-up of 15 months. There are more data available in Japan, but the experience in the United States, I think, is still evolving. That is one of the things that we really want to study. I think there might be a complementary role for SRS in combination with RFA.

Dr Harold Urschel (Dallas, Tex). Thank you, Dr Pennathur. That was a very nice presentation. How about making a comment on the Cyberknife, the linear accelerator with the robot that actually treats the same kind of lesions, including now, hopefully, central lesions and comparing it with the possibility—we are in the Stanford, Pittsburgh, Dallas, Georgetown, and Boston group-of trying to find these cases with what we think is a better approach. What do you think about that?

Dr Pennathur. Thank you for your comments. I think Cyberknife is one of the 3 available pieces of equipment by which we can deliver SRS and is a frameless system. In the United States I think we have only a fair experience with stereotactic surgery. One 
point I want to mention that Dr Low alluded to earlier was the need for thoracic procedures, which is also required for SRS; that is, you have to place fiducials to mark and track the tumor for SRS. When placing fiducials, you need typically about 3 to track the tumor. In our institution we place these fiducials with CT guidance, and we do the treatment planning with radiation oncology before SRS also. The fiducials are not benign. We need to place the coaxial needle about 3 times or so in the lung and place the fiducials. I cannot remember the percentages, but there is a significant incidence of pneumothorax with the placement of fiducials. For the more central lesions, we might be able to perform the fiducial placement with the bronchoscope in the future and that might avoid some of the pneumothorax complications, but at the moment, we are doing percutaneous CT-guided fiducials, which do cause pneumothoraces. I think in this area the multicenter trial we have with Dr Urschel, Dr Whyte, and Georgetown-Boston is going to be very important in answering some of the questions in terms of SRS, patient selection, and evaluation of long-term results. Unlike RFA, in which the complications you see are within the first month typically, with SRS, particularly for central lesions, you see complications, such as bronchial stenosis and so forth, months and sometimes more than a year later down the road because of the effects of radiation with fibrosis. Therefore I think more long-term studies are required for us to really define the role of which patient might be more suitable for SRS versus RFA and whether there is a complementary role to using both of them.

Dr Eric Vallieres (Seattle, Wash). I appreciated your study and Don's and everyone else's comments. I think in the future when you are going to compare these various modalities you are going to have to include cost. Radiation tools are not free. I think you are going to have to include hospital stay and how many treatments they need. This is a 1-day treatment. If your patient does not have any complications, when do you send him home? What is your hospitalization time on these?

Dr Pennathur. Thank you for your comments and questions. The median hospital stay for us was 3 days. This patient group was elderly, with multiple comorbidities, and we admitted almost all of them. There is usually a zone of inflammation after the RFA, and some have a pigtail catheter for the pneumothorax. Typically, we admit them for a couple of days or so and then send them home.

Dr Vallieres. I think all of these factors are going to have to weigh in because you know most of these folks die of their noncancer; that is, they have a noncancer death. They die of their comorbidities, and therefore really what it is going to come down to is cost and how quickly you can deliver the treatment and get them home.

Dr Pennathur. I think that is an excellent point. Costwise I think the Cyberknife and the SRS are quite expensive, and the institution has to invest the money in that. When you place fiducials and if the patient has a pneumothorax, we admit them, and that requires a few days' hospitalization; then the patient comes back for the radiosurgery, which is usually done as an outpatient. With SRS, however, typically it is only 3 days as opposed to the 6 weeks of treatment that is required for conventional radiation.

I thank the Association for the privilege of presenting this paper. 